\title{
Diseño de productos turísticos rurales utilizando los recursos naturales, patrimoniales y culturales
}

\author{
DESIGN OF TOURIST PRODUCTS RURAL USING NATURAL, CULTURAL AND HERITAGE RESOURCES
}

\author{
Luis Fernando Figueroa González ${ }^{16}$ \\ Ríos Rubiano Erick Raúl ${ }^{17}$ \\ Valencia Becerra Denice ${ }^{18}$
}

\section{Resumen}

El turismo rural sustentable representa una alternativa para el desarrollo económico, social y ecológico de las comunidades rurales, para lo cual es necesario desarrollar productos y servicios turísticos apropiados. El objetivo de esta investigación consistió en identificar los recursos pasivos de una comunidad para el desarrollo de productos turísticos rurales sustentables. Mediante la revisión de literatura se explicaron los conceptos del turismo alternativo así como el turismo rural, idenficandose los diversos recursos pasivos con que cuenta una comunidad y que son factibles de ofertar como atractivos turísticos. Mediante esta investigación conceptual se pudieron identificar toda una serie de recursos naturales factibles de aprovechar y comercializar como productos turísticos rurales. Concluyendo que para el desarrollo de producto y servicios turísticos rurales sustentables en relación con los recursos pasivos de una comunidad, solo será posible con el trabajo coordinado de los principales actores habitantes, gobiernos, empresarios.

Palabras clave: Turismo rural, sustentabilidad, turismo alternativo.

\begin{abstract}
Sustainable rural tourism represents an alternative to the economic, social and ecological development in rural communities, for which it is necessary to develop tourism products and services appropriate. The objective of this research consisted of identifying passive resources of a community for the development of sustainable rural tourism products. By reviewing the literature explained the concepts of alternative tourism and rural tourism, identified various passive resource that has a community and that they are feasible to offer as tourist attractions. A range of feasible to exploit and market as rural tourism products natural resources could be identified through this conceptual research. Concluding that you for the development of product and sustainable rural tourism services in relation to the passive resources of a community, only it will be possible with the coordinated work of inhabitants main stakeholders, Governments and entrepreneurs.
\end{abstract}

Key words: rural tourism, sustainability, alternative tourism.

\footnotetext{
${ }^{16}$ Departamento de Contabilidad, Universidad de Sonora, ffigueroa@eca.uson.mx

${ }^{17}$ Estudiante de la licenciatura en mercadotecnia. Universidad de Sonora.

${ }^{18}$ Estudiante de la licenciatura en mercadotecnia. Universidad de Sonora.
} 


\section{Introducción}

El turismo es una actividad económica que proporciona productos y servicios a los visitantes, y comprende una serie de aspectos como la estancia, el conocimiento de culturas, historia y recursos naturales cuyo objetivo es el esparcimiento humano. Asimismo, es una industria que va en rápido incremento en todas las economías, ya que es un factor de crecimiento al generar flujos continuos de ingresos (Rogel et al., 2011).

La actividad del turismo en las últimas décadas ha ido adquiriendo una gran importancia y con una tendencia de crecimiento para los próximos años, pues ha pasado de ser un fenómeno natural donde se involucra el aspecto social pero sobre todo el económico, a ser considerado un fenómeno estructural que influye en la economía internacional en muchos países, regiones y localidades (Barroso y Flores, 2006). Por lo tanto para el siglo XXI el turismo estará presente en numerosos países que antes no lo consideraban una fuente de ingreso importante para sus economías, pero que ahora invierten en la promoción de sus recursos turísticos para lograr un flujo constante de visitantes (Rangifo, 2008).

El turismo en México representa el 9\% del PIB, y contribuye a la generación de más de 7.5 millones de empleos, y es considerado dentro de los 10 destinos turísticos más importantes del mundo (Guevara, 2011). Estos resultados tienen su origen de la política pública planteada en el primer Plan Nacional de Desarrollo Turístico elaborado por el Gobierno Mexicano en los años 60's y fue detonante de varios destinos turísticos como Cancún, Los Cabos, y Loreto entre otros (Benseny, 2007).

El turismo alternativo representa una opción distinta al turismo tradicional de sol y playa, ya que brinda la oportunidad de explorar nuevos destinos turísticos, y es considerado una alternativa del uso sustentable de los recursos, y a la vez permite alternar e interactuar con una población.
A su vez está integrado por otras modalidades como el turismo rural, turismo cultural, turismo de convenciones, turismo de aventura, entre otros. Asimismo se consideran cuatro dimensiones en el que se desarrolla: espacial, ambiental, económica y social, siendo en esta última donde el turismo rural está considerado (Pérez et al., 2010).

A nivel internacional, el turismo rural es una actividad importante especialmente en países subdesarrollados, pues permite incrementar sus economías y disminuir la pobreza en esas zonas, por lo que se han implementado programas para su desarrollo a través de organismos como el Banco Mundial, el Fondo Interamericano de Desarrollo Agrícola entre otros (Pérez et al., 2010). Y se define como una forma alternativa de viaje, que lleva a las personas a lugares rurales, con la idea de conocer el campo y practicar actividades y deportes en contacto con la naturaleza, presentándose en diversas variantes como el ecoturismo, agroturismo, turismo de naturaleza, turismos de aventura, y que envuelve actividades como caza, pesca, visitas culturales e históricas entre otras (Millán et al., 2006; Solsona, 2006).

En este sentido el turismo rural, genera importantes ingresos complementarios a las actividades agrarias, además de proporcionar beneficios para las empresas relacionadas con esta actividad (alojamientos), así como para las relacionadas de una manera indirecta como son gasolineras, tiendas, restaurantes, entre otros (Diéguez et al., 2009).

En el turismo rural se presenta una desventaja que es su estacionalidad la cual obstaculiza el ingreso constante adicional en las comunidades, por lo que es recomendable diversificar los productos y servicios en las comunidades, con el objetivo disminuir la estacionalidad del turismo y permitir un flujo más continuo de ingresos adicionales (Pérez et al., 2010). 
Para poder cumplir con lo anterior, es importante crear la estructuración de una oferta turística local que se considere a mediano y largo plazo y que se convierta en un factor de desarrollo, aprovechando los recursos potenciales con que cuenta una zona rural, cuidando mantener un equilibrio sostenido con la naturaleza, pero que permita una ventaja competitiva para la comunidad (Mazaro y Varzin, 2008).

Por lo tanto, se debe confirmar que una zona rural posee recursos potenciales para el desarrollo del turismo que permita justificar una inversión, haciendo una evaluación rigurosa de los recursos para considerar la viabilidad de ofertarlo, ya sea en forma natural o haciendo mejoras y adaptaciones para crear el producto adecuado que llame la atención del visitante (Bringas, 2008).

Considerando lo anteriormente expuesto, el objetivo de esta investigación es identificar aquellos recursos naturales, culturales y patrimoniales de una comunidad que puedan ser factibles de desarrollar y comercializar respetando el medio ambiente.

\section{Revisión de literatura}

\section{Turismo alternativo}

Las necesidades actuales de las personas en cuanto a viajar se refiere, se están enfocando a romper con lo rutinario o tradicional para salir de la monotonía del turismo tradicional, esta situación ha impulsado que comunidades rurales incorporen atractivos naturales y actividades de esparcimiento a través de actividades locales con el fin de satisfacer las necesidades de las personas de experimentar nuevas formas de hacer turismo (López-Guzmán y Cañizares, 2009). Para lograr lo anterior, es de vital importancia el apoyo de los residentes de las comunidades que muestren una actitud abierta y hospitalaria, así como la armonía entre los intereses de las personas que interviene para lograr el desarrollo del turismo con éxito (Royo y Ruiz, 2009).
El Turismo Alternativo (TA) ha sido nombrado de diversas formas como son: Turismo Responsable, Nuevo Turismo, Turismo blando y Turismo de Bajo Impacto, por lo que ha sido interpretado de diversas formas como por ejemplo: elemento contrario y sustituto del turismo de masas, así como, nuevo nicho de mercado con demandas particulares de productos y servicios de nuevos consumidores (Higgins-DesbioUes, 2008).

En su acepción más general, el Turismo Alternativo se puede definir de forma global como el conjunto de modalidades turísticas que tienen por objetivo ser respetuosos con los valores naturales, sociales y comunitarios, permitiéndoles además, a los anfitriones como a los huéspedes, disfrutar de una interacción positiva y del compartimiento de experiencias (González et al., 2007). En vista de lo anterior se puede afirmar que el turismo alternativo es consistente con la naturaleza, con el aspecto social y con los valores de una comunidad, permitiendo a la vez que tanto anfitriones como huéspedes disfruten en forma positiva interactuando y compartiendo experiencias a través de los productos y servicios que brinda la comunidad al visitante (Rami, 2009).

De acuerdo con Rogel et al., 2011, se puede establecer una clasificación del turismo la cual abarca dos conceptos: Turismo Convencional y Turismo Alternativo el cual a su vez considera el Ecoturismo, el Turismo de Aventura y el Turismo Rural. Por lo que es conveniente, realizar una segmentación del turismo alternativo fundamentado en el interés y actividades que el turista tiene y busca al estar en contacto con la naturaleza. En este sentido la SECTUR dividió al turismo alternativo en tres segmentos los cuales definió de la siguiente manera

Ecoturismo; son viajes para apreciar y conocer la naturaleza a través de la observación de la flora, la fauna y fenómenos de la naturaleza, el senderismo y la educación ambiental. 
Turismo de aventura: son las actividades con relación directa con la naturaleza en tres ambientes: aire (vuelo en globo, paracaidismo, ala delta); agua (buceo, pesca recreativa, descenso en río); tierra (rappel, ciclismo de montaña, cabalgata, montañismo).

Turismo rural: Los viajes que tienen como fin realizar actividades de convivencia e interacción con una comunidad rural, como son: el etnoturismo, agroturismo, fotografía rural, talleres artesanales, talleres gastronómicos entre otras.

\section{Turismo rural}

El turismo rural es una actividad económica que desarrolla una serie de servicios y productos que generan ingresos adicionales, por lo que es una opción complementaria a la actividad agrícola tradicional, en virtud de que promueve el desarrollo y aprovechamiento de productos turísticos alternativos, por lo que se puede definir como: "una gama de actividades y amenidades provistas por campesinos y personas rurales para atraer turistas, y así generar un ingreso extra” (Pérez, 2010, p. 5). En una forma más extensa el turismo rural incluye el concepto vivo de la historia, costumbres rurales, el folclor, tradiciones familiares, valores, y herencia patrimonial (MacDonald y Jooliffe, 2003).

Sin embargo a pesar de que el desarrollo del turismo en el ámbito rural tiene muchas bondades, su crecimiento debe ir acompañado de ciertos controles en virtud de que una fuerte demanda puede ocasionar impactos negativos al medio ambiente, y en las comunidades donde se lleva a cabo, como es la acumulación de basura, la contaminación del aire entre otros, por lo que los planificadores deben mantener un equilibrio entre la demanda y la oferta turística derivado del incremento en el gusto de la personas por realizar una turismo diferente al tradicional y poder estar en contacto con la naturaleza. Por lo que la participación de los miembros de las comunidades resulta de vital importancia en el manejo de las áreas naturales para lograr una sustentabilidad de los recursos con que se cuenta (Saarinen, 2006; Nolte, 2008; Bruyer et al., 2009; Touraya y Junga, 2010).
Por lo tanto el desarrollo de esta actividad económica para que se pueda realizar en forma óptima tiene que enfrentar una serie de retos o desafíos (figura 1) como son: Las experiencias rurales que los nuevos turistas buscan, y las cuales se deben de desarrollar y mantener; Desarrollo Regional que es la administración de los recursos para generar oportunidades y conocer las limitaciones; Equilibrio el cual el TR debe considerar en las relaciones de los involucrados en esta actividad (visitantes, anfitriones, comerciantes, autoridades, etc.); Ambiente sustentable donde se deben desarrollar regla, normas, políticas, relacionadas con el cuidado del ambiente natural; Integración, el TR se debe considerar en los planes de desarrollo tanto estatales como nacionales; y Desarrollo de negocios, en este sentido en la promoción de negocios rurales, el TR enfrenta una serie de obstáculos como son: pobres inversiones, escasas o nulas habilidades o recursos insuficientes para comercializar, falta de capacitación a las personas y la falta de cooperación con otras empresas turísticas (Sharpley, 2007).

Figura 1. Desafíos del Turismo Rural.

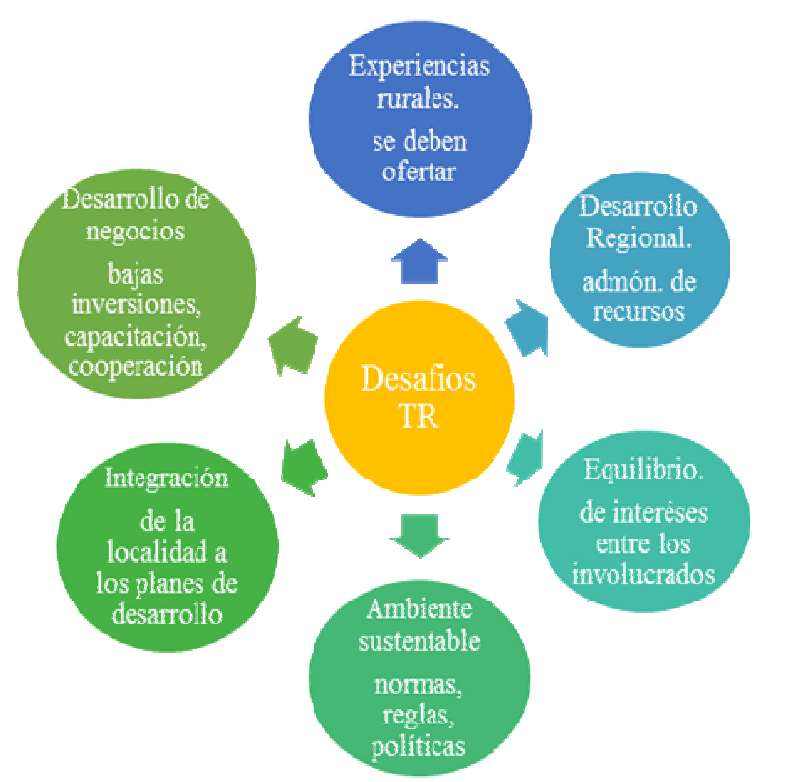

Fuente: Elaboración propia con información de Sharpley, 2007. 
Aunado a lo anterior, la creciente conciencia sobre la importancia del turismo sustentable, en el sentido de que su crecimiento no podía continuar sin hacer frente a los impactos de este sobre el medio ambiente ha sido clave para considerar la sustentabilidad y el turismo sustentable en el diseño de cualquier plan para desarrollar el turismo rural (Touraya, y Junga, 2010).

\section{El Turismo Rural y la Sustentabilidad}

La idea del turismo sustentable, es que contempla el reconocimiento de los impactos negativos, y la necesidad de manejarlos para lograr la meta de un desarrollo sostenible (Saarinen, 2006). En este sentido la participación de la comunidad en el manejo de las áreas naturales es de vital importancia, donde el turismo sustentable es considerado como una estrategia para la conservación de estas áreas (Bruyer et al., 2009).

Aunado a lo anterior, la creciente conciencia sobre la importancia del turismo sustentable, en el sentido de que su crecimiento no podía continuar sin hacer frente a los impactos de este sobre el medio ambiente ha sido clave para considerar la sustentabilidad en el turismo para el diseño de cualquier plan para desarrollar el turismo rural (Touraya, y Junga, 2010).

Es por esto que el Turismo Rural Sustentable (TRS) tiene como objetivo minimizar el daño ambiental y cultural y contribuir al crecimiento económico, pero manteniendo un equilibrio entre el crecimiento y la conservación del medio ambiente a través de una buena relación entre los lugares de destino y sus habitantes, los turistas y los proveedores de productos y servicios (Lane, 2005). Por lo anteriormente expuesto, se puede decir que todas las actividades turísticas deben ser sustentables en todas sus dimensiones, y por lo tanto el turismo rural no solo no es la excepción, sino que además se convierte en el principal impulsor del desarrollo sustentable (Pinilla, 2009). Esto en parte derivado del crecimiento de la demanda del turismo de naturaleza, el cual está provocando cambios en los patrones de conducta del turista (Nolte, 2008).

\section{Identificación de los recursos pasivos}

Los recursos naturales que se utilizan para el desarrollo del TR normalmente son conocidos como Capital del campo, los cuales son empleados en la generación de negocios de turismo en diferentes proporciones con el fin de atraer turistas a su comunidad y suministrar las experiencias naturales que ellos buscan (Garrod et al., 2006). Asimismo los recursos patrimoniales y culturales que al igual que los naturales son conocidos como recursos pasivos, pueden ser factibles de convertirse en productos turísticos rurales sustentables y comercializarse tabla 1(García, 2005):

Tabla 1. Productos rurales de recursos pasivos.

\begin{tabular}{ccc}
\hline $\begin{array}{c}\text { Recursos } \\
\text { naturales }\end{array}$ & $\begin{array}{c}\text { Patrimonio } \\
\text { histórico }\end{array}$ & $\begin{array}{c}\text { Manifestacione } \\
\text { s } \\
\text { culturales }\end{array}$ \\
\hline Paisajes & $\begin{array}{c}\text { Monumentos } \\
\text { Arquitectura } \\
\text { popular }\end{array}$ & $\begin{array}{c}\text { Folclore } \\
\text { Gastronomía }\end{array}$ \\
$\begin{array}{c}\text { Parques } \\
\text { naturales } \\
\text { Áreas de } \\
\text { recreo } \\
\text { Camillo, viejas } \\
\text { edificaciones }\end{array}$ & $\begin{array}{c}\text { Celebraciones } \\
\text { religiosas }\end{array}$ \\
$\begin{array}{c}\text { Escultura } \\
\text { Fauna }\end{array}$ & Artesanías & Escultura \\
& Yacimientos & Ferias \\
Flora & & $\begin{array}{c}\text { Costumbales } \\
\text { tradiciones } \\
\text { populares }\end{array}$ \\
\hline
\end{tabular}

Fuente: García, 2005. 


\section{Productos turísticos rurales}

El TRS aprovecha los recursos rurales y naturales, combinándolos y organizándolos en productos turísticos que generan una variedad de actividades entre las que se encuentran: el Turismo Cultural que se basa en manifestaciones culturales de una comunidad, Turismo Deportivo actividades deportivas que se realizan en contacto con la naturaleza como es la cacería, Turismo Gastronómico el cual contempla las riquezas gastronómicas de cada destino, Turismo de Salud que implica actividades relacionadas con el conocimiento de plantas medicinales y el Turismo Educativo que abarca experiencias educativas sobre el conocimiento de costumbre de los espacios rurales (Pérez, 2010), el Agroturismo que se refiere a experiencias con las actividades cotidianas de los campesinos (Santinia et al., 2011), el Ecoturismo el cual considera actividades de esparcimiento en el medio rural con sentido responsable del medio ambiente (Pérez et al., 2010), Turismo de Aventura es un tipo de turismo activo con deportes de aventura en los medios rurales naturales considerando el agua, el viento y la tierra (Rogel et al., 2011).

Los productos turísticos rurales se complementan con una serie de servicios básicos que agregan valor añadido al producto como es el alojamiento, comercios, productos de la tierra, gastronomía y servicios de actividades complementarias, éstos deben ser considerados en cualquier destino turístico rural, formando parte del producto llamado turismo rural, en la tabla 2 se presenta un desglose de estos elementos (García, 2005).
Tabla 2. Elementos del producto Turismo Rural.

\begin{tabular}{|c|c|}
\hline Producto & Recurso \\
\hline $\begin{array}{l}\text { - Naturaleza } \\
\text { (recurso pasivo) }\end{array}$ & - $\quad$ Recurso turístico \\
\hline - Cultura (recurso & Recurso turístico \\
\hline pasivo) & _ $\quad$ Recurso turístico \\
\hline $\begin{array}{l}\text { - Edificaciones } \\
\text { antiguas (recurso }\end{array}$ & - $\quad$ Recurso turístico \\
\hline pasivo) & Actividades \\
\hline $\begin{array}{l}\text { - Turismo activo } \\
\text { (recurso activo) }\end{array}$ & Actividades \\
\hline - Gastronomía & Servicios \\
\hline - Rutas & - $\quad$ Servicios \\
\hline $\begin{array}{l}\text { - Alojamiento } \\
\text { - Información }\end{array}$ & - $\quad$ Servicios \\
\hline - Comunicaciones & - $\quad$ Servicios \\
\hline $\begin{array}{l}\text { - Folletos y } \\
\text { catálogos }\end{array}$ & - $\quad$ Servicios \\
\hline - Internet & - $\quad$ Servicios \\
\hline $\begin{array}{l}\text { - Servicios } \\
\text { financieros }\end{array}$ & - $\quad$ Servicios \\
\hline
\end{tabular}

Fuente: García, 2005.

\section{Resultados}

Con la presente investigación de carácter conceptual a través de la revisión de literatura relacionada con el tema, se pudo determinar o identificar aquellos recursos pasivos con que cuenta la mayoría de las comunidades en México que pueden ser factibles de transformar en productos turísticos rurales sustentables.

Dentro de los recursos naturales de una comunidad se pueden desarrollar una serie de actividades en contacto con la naturaleza que se pueden ofertar a los visitantes como pueden ser: senderismo, turismo de aventura, turismo deportivo, paisajes, flora y fauna entre otros.

Para el aprovechamiento del patrimonio histórico se deben promocionar, los edificios arquitectónicos, las ruinas, haciendas, edificaciones antiguas, yacimientos, monumentos, esculturas, etc. 
Las manifestaciones culturales como recurso pasivo también presentan toda una gama de actividades que pueden ofertarse a los visitantes como pueden ser: danzas tradicionales dentro del folclore, rutas de comida en lo relacionado con la gastronomía, las celebraciones religiosas, las ferias.

Dependiendo de las características de cada comunidad se pueden desarrollar toda una serie de productos turísticos rurales sustentable que pueden ser factibles de ofertarse a los turistas con el fin de que las comunidades sean visitadas con regularidad y no solo de forma estacional.

\section{Conclusiones}

El turismo rural sustentable al desarrollarse en forma correcta y debidamente planeada, trae consigo toda una serie de beneficios para los habitantes de las comunidades como es la diversificación del empleo, revalora el patrimonio cultural, patrimonial y ambiental, se comercializan productos locales, y genera incremento adicional en los ingresos de los habitantes.

El desarrollo del turismo rural sustentable representa una alternativa viable para el desarrollo de las comunidades atendiendo la demanda de productos y servicios turísticos rurales, motivo por el cual esta actividad ha estado siendo adoptada por diversos países los cuales en su mayoría la consideran como parte de sus planes de desarrollo con el fin de que las personas no dependan únicamente de las actividades agrícolas.

Pero el desarrollo de producto y servicios turísticos rurales sustentables en relación con los recursos pasivos de una comunidad, solo será posible cuando exista una real integración de los diversos actores que pueden intervenir para el desarrollo de esta actividad como son los habitantes de las comunidades, proveedores de servicios turísticos, empresarios y gobiernos.

Esta integración de los actores de esta actividad resulta de vital importancia, tomando en cuenta que en la mayoría de las comunidades no cuentan o tienen pobres inversiones, escasas o nulas habilidades o recursos insuficientes para comercializar, falta de capacitación a las personas y la falta de cooperación con otras empresas turísticas.

\section{Referencias}

Barroso, M., y, Flores, D. (2006). La competitividad internacional de los destinos turísticos: del enfoque macroeconómico al enfoque estratégico. Cuadernos de Turismo, enero-junio, 7-24.

Benseny, G. (2007). El turismo en México apreciaciones sobre el turismo en espacio litoral. Aportes y Transferencias, 13-34.

Bringas, O. 2008. El turismo Rural. Revista Universidad De Sonora.

http://www.revistauniversidad.uson.mx/revistas/21El\%20turismo\%20rural.pdf recuperado el 17 de Octubre de 2012.

Bruyere, B. L., Beh, A. W., y Lelengula, G. (2009). Differences in perceptions of communication, tourism benefits, and management issues in a protected area of rural Kenya. Environmental Management, 43, 1, 49-59.

Diéguez, M., Gueimonde, A., y Sinde-Cantorna, A. I. (2009). Turismo rural como estrategia de diversificación: factores determinantes y resultados en Galicia. (Spanish). Cuadernos De Gestión, 9 (2), 31-53.

García, B. (2005). Características diferenciales del producto turismo rural. Cuadernos deTurismo, enerojunio, 113-133.

Garrod, B., Wornell, R. y Youell, R. (2006). Reconceptualising rural resources as countryside capital:The case of rural tourism. Journal of Rural Studies 22 (2006) 117-128.

González, D., Pereira, C., y Companioni, C. (2007). Vía para la diversificación de un destino Turismo Alternativo. (Spanish). Retos Turísticos, 6(3), 27-34.

Higgins-Desbiolles, F. (2008). Justice Tourism and Alternative Globalisation. Journal of Sustainable Tourism, 16(3), 345-364. 
Lane, B. (2005). Sustainable Rural Tourism Strategies: A tool for development and conservation. RIAT Revista Interamericana de Ambiente y Turismo. Volumen 1, número 1 Sustainable Tourism, 16(3), 345-364.

López-Guzmán, T. y Cañizares, s. (2009). Desarrollo socioeconómico de las zonas rurales con base en el turismo comunitario. Un estudio de caso en Nicaragua. (Spanish). Cuadernos De Desarrollo Rural, (62), 81-97.

MacDonald, R. y Jooliffe, L. (2003). Cultural rural tourism evidence from Canada. Annals of Tourism Research, Vol. 30, No. 2, pp. 307-322, 2003.

Mazaro, R., y Varzin, G. (2008). Modelos de Competitividad para Destinos Turísticos en el Marco de la Sostenibilidad. (Spanish). RAC - Revista De Administração Contemporânea, 12(3), 789-809.

Millán, M., López-Guzmán, T., y, Agudo, E. (2006). El turismo rural como agente económico: desarrollo y distribución de la renta en la zona de Priego de Córdoba. CIRIEC-España, Revista de Economía Pública, Social y Cooperativa, agosto, 167-192.

Nolte, B. (2008). Sustainable tourism development in cross-border biosphere reserves of Central and Eastern Europe. In Cross-border Governance and Sustainable Spatial Development (pp.147-160). Springer Berlin Heidelberg.

Pérez, A., Juárez, J., Ramírez, B., y, Cesar, F. (2010). Turismo rural y empleo rural no agrícola en la Sierra Nororiente del estado de Puebla: caso red de Turismo Alternativo Totaltikpak, A.C. Investigaciones Geográficas (Mx), Abril, 57-71.

Pérez, S. (2010). El valor estratégico del turismo rural como alternativa sostenible de desarrollo territorial rural. Agronomía Colombiana, Septiembre-Diciembre, 507-513.

Pinilla, H. (2009). Entre lo ideal y lo real; ¿̇los cambios en los enfoques propuestos de turismo rural sostenible desde la Organización de las Naciones Unidas contribuirían al desarrollo rural territorial? (Spanish). Cuadernos De Desarrollo Rural, (62), 145-171.
Rami. I. (2009): Alternative Tourism: Can the Segregation Wall in Bethlehem be a Tourist Attraction?, Tourism and Hospitality Planning \& Development, 6:3, 247-254.

Rengifo, J. (2008). Un segmento del turismo internacional en auge: el turismo de caza.

Rogel Fajardo, Isidro; Rojas Lopez, Aracely; Ortega Vega, Samantha Yajaira. (2011). El turismo alternativo como estrategia de conservación de la reserva de la biosfera de la mariposa monarca (2008-2010). Quivera, Julio-Diciembre, 115-133.Cuadernos de Turismo, JulioDiciembre, 187-210.

Royo, M. y Ruiz, M. (2009). Actitud del residente hacia el turismo y el visitante: factores determinantes en el turismo y excursionismo rural-cultural. Cuadernos de Turismo, Enero-Junio, 217-236.

Saarinen, J. (2006). Traditions of sustainability in tourism studies. Annals of Tourism Research, Vol. 33, No. 4, pp. 1121-1140.

Santinia, C., Cavicchib, A., y Canavaric, M., (2011). The Risk ${ }^{\mathrm{TM}}$ strategic game of rural tourism: how sensory analysis can help in achieving a sustainable competitive advantage. Food, Agriculture and tourism. pp 161-179.

Sharpley, R. (2007): Flagship Attractions and Sustainable Rural Tourism Development: The Case of the Alnwick Garden, England, Journal of Sustainable Tourism, 15:2, 125-143.

Solsona, J. (2006). El turismo rural en Europa. Aportes y Transferencias, Sin mes, 25-35.

Touraya, K. y Junga, T. (2010). Exploratory Study on Contributions of ICTs to Sustainable Tourism Development in Manchester. Information and Communication Technologies in Tourism p.p. 493-505. 